\title{
Sun-Yung Alice Chang and Geometric Analysis
}

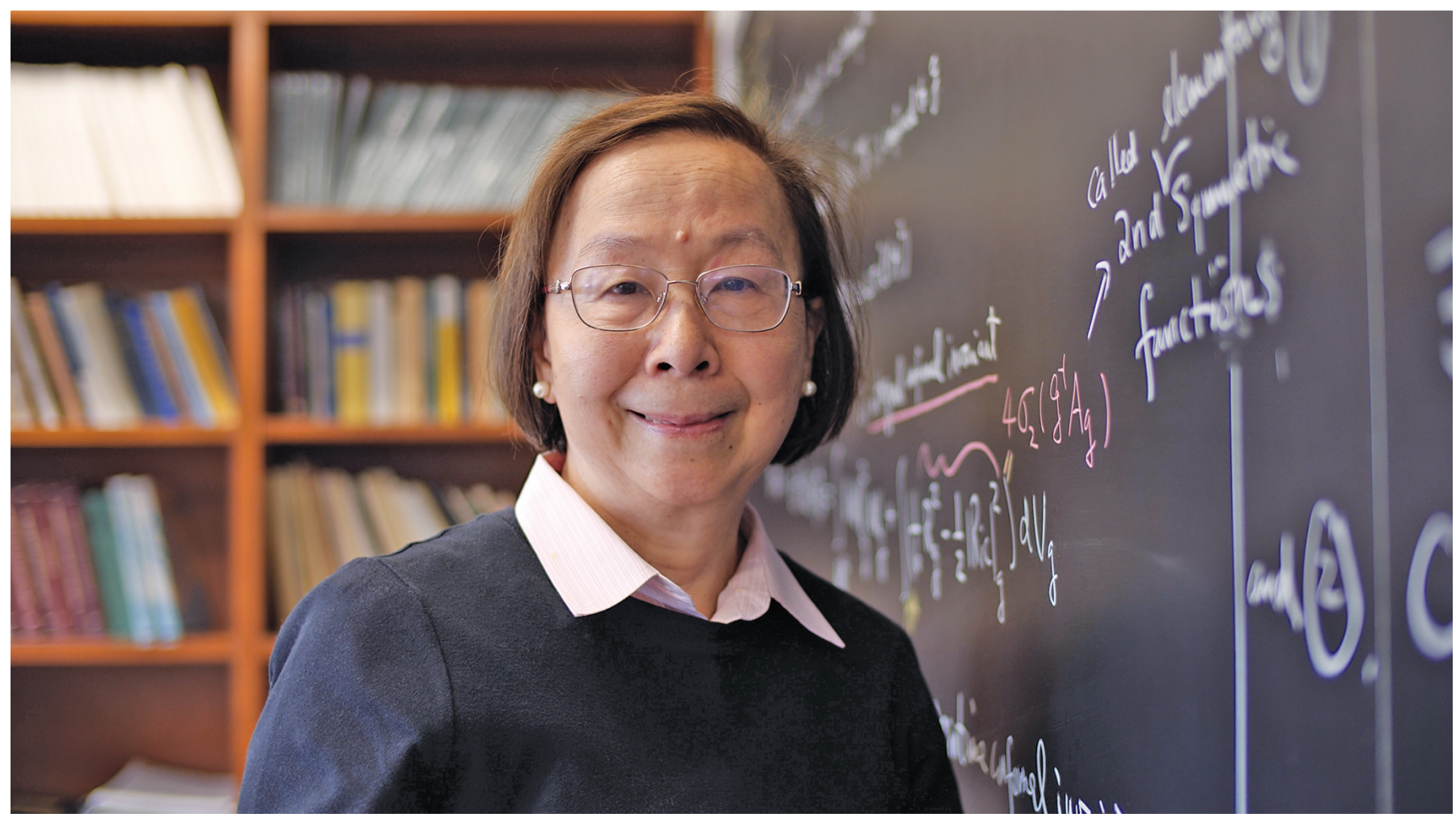

\section{Matthew J. Gursky and Yi Wang}

Sun-Yung Alice Chang was born in Xi'an, China, in 1948, and grew up in Taiwan. She did her undergraduate work at National Taiwan University, graduating in 1970 . Her experience at NTU as one of a group of women in the same class who majored in mathematics, finished near the top of their class, and went on to enjoy successful careers was the subject of the award-winning documentary Girls Who Fell in Love with Math, released in 2017. Alice came to the United States for graduate studies, and received her $\mathrm{PhD}$ in 1974 from the University of California, Berkeley, under the supervision of Donald Sarason.

Matthew J. Gursky is a professor of mathematics at the University of Notre Dame. His email address is mgursky@nd.edu.

$Y i$ Wang is an assistant professor of mathematics at Johns Hopkins University. Her email address is ywang@math. jhu.edu.

Communicated by Notices Associate Editor Chikako Mese.

For permission to reprint this article, please contact:

reprint-permission@ams.org.

DOI: https://doi.org/10.1090/noti2037
Alice's early work was in the field of classical harmonic analysis. Sarason had been interested in a question posed by Douglas in the late 1960s about the boundary behavior of bounded analytic functions on the unit disc, which he called "The Douglas Problem." One of Alice's first publications, "A characterization of Douglas subalgebras" [4], provided a key part of the solution, and combined with the work of Marshall [20] gave a complete resolution. Both papers appeared in Acta Mathematica in 1976, and the combined result is now widely referred to as the Chang-Marshall theorem. Over the next decade Alice continued making important contributions to the theory of function algebras, including two papers that appeared in the Annals of Mathematics: "Carleson measure on the bidisc" [5] and "On a continuous version of duality of $H^{1}$ with $\mathrm{BMO}$ on the bi-disc" [3], the latter coauthored with Robert Fefferman.

In the mid-1980s her research went in a new direction, and she was increasingly attracted to problems that were 
influenced by geometry. In this article we will give an overview of Alice's contributions to geometric analysis, beginning with her work with Carleson on the critical case of the classical Sobolev embedding theorem. This result, surprising in itself, anticipated several developments in her subsequent research.

\section{The Limiting Case of the Classical Sobolev Inequalities}

The classical Sobolev inequality states that for $1<p<n$, there is a constant $C(n, p)$ such that if $u \in C^{\infty}\left(\mathbb{R}^{n}\right)$, then

$$
\left(\int_{\mathbb{R}^{n}}|u|^{\frac{n p}{n-p}} d x\right)^{\frac{n-p}{n}} \leq C(n, p) \int_{\mathbb{R}^{n}}|\nabla u|^{p} d x .
$$

The optimal constant in this inequality, which we will denote by $C_{n, p}\left(\mathbb{R}^{n}\right)$, is called the Sobolev constant. If $W^{1, p}\left(\mathbb{R}^{n}\right)$ is the Sobolev space of functions in $L^{p}\left(\mathbb{R}^{n}\right)$ with (distributional) derivative in $L^{p}$, then (1) implies that for $1<p<n$ there is a continuous embedding $W^{1, p}\left(\mathbb{R}^{n}\right) \hookrightarrow L^{\frac{n p}{n-p}}\left(\mathbb{R}^{n}\right)$. Moreover, the norm of the embedding is given by the Sobolev constant. When $p=1$ the embedding is still valid, and the optimal constant is the isoperimetric constant.

The precise value of the Sobolev constant $C_{n, p}\left(\mathbb{R}^{n}\right)$ was determined by Aubin and Talenti, who also showed that equality holds in (1) with $C(n, p)=C_{n, p}\left(\mathbb{R}^{n}\right)$ if and only if $u$ is given by

$$
u_{a, b, x_{0}}(x)=\left\{a+b\left|x-x_{0}\right|^{p /(p-1)}\right\}^{1-n / p},
$$

where $x_{0} \in \mathbb{R}^{n}$ and $a, b>0$ are constants.

Now suppose $\Omega \subset \mathbb{R}^{n}$ is a bounded domain. We can define the Sobolev space $W^{1, p}(\Omega)$ as we did for $\mathbb{R}^{n}$, and we also let $W_{0}^{1, p}(\Omega)$ denote the closure of $C_{0}^{\infty}(\Omega)$ in $W^{1, p}(\Omega)$. If $C_{n, p}(\Omega)$ denotes the optimal constant in (1) for all $u \in W_{0}^{1, p}(\Omega)$, then it turns out that $C_{n, p}(\Omega)=C_{n, p}\left(\mathbb{R}^{n}\right)$. It may seem surprising that $C_{n, p}(\Omega)$ is independent of $\Omega$, but this fact reflects an important property of the extremal functions in (2). Namely, if we take $x_{0} \in \Omega$, then as $a \rightarrow 0$ and $b \rightarrow \infty$, the function $u_{a, b, x_{0}}(x)$ will concentrate near $x_{0}$; i.e., $u_{a, b, x_{0}}\left(x_{0}\right) \rightarrow \infty$ while $u_{a, b, x_{0}}(x) \rightarrow 0$ for all $x \neq x_{0}$. By multiplying by a cut-off function we can easily construct functions $\tilde{u}_{a, b} \in C_{0}^{\infty}(\Omega)$ so that

$$
\frac{\left(\int_{\Omega}\left|\tilde{u}_{a, b}\right|^{\frac{n p}{n-p}} d x\right)^{\frac{n-p}{n}}}{\int_{\Omega}\left|\nabla \tilde{u}_{a, b}\right|^{p} d x} \rightarrow C_{n, p}\left(\mathbb{R}^{n}\right),
$$

as $a \rightarrow 0, b \rightarrow \infty$.

Another consequence of this construction is that there are sequences of functions that are bounded in $W^{1, p}(\Omega)$, but which have no subsequence that converges in $L^{\frac{n p}{n-p}}(\Omega)$ -indeed, the functions $\tilde{u}_{a, b}$ converge almost everywhere to zero as $a \rightarrow 0, b \rightarrow \infty$. A related but less obvious fact is that the optimal constant $C_{n, p}(\Omega)$ is not attained. If it were attained by some function $v_{\Omega} \in W_{0}^{1, p}(\Omega)$, then it is not difficult to see that $v_{\Omega}$ would be an extremal for the inequality (1) as well. This would mean that $v_{\Omega}$ would be of the form (2), but these functions are not compactly supported.

A natural question, which also turns out to have important geometric consequences, is what happens in the limiting case $p=n$. Notice that as $p \nearrow n, \frac{n p}{n-p} \nearrow \infty$. However, it is easy to see that there are functions in $W_{0}^{1, n}(\Omega)$ that are not in $L^{\infty}(\Omega)$ : If $x_{0} \in \Omega$, just take $u(x)=\log \log \frac{1}{\left|x-x_{0}\right|}$ (and multiply by a cut-off function). Nevertheless, when $p=n$ there is a beautiful inequality proved by Neil Trudinger in 1967 [22]:

Theorem 1.1. Let $\Omega \subset \mathbb{R}^{n}$. There are constants $\beta=$ $\beta(n), C_{0}=C_{0}(\Omega)$ with the following property: If $u \in W_{0}^{1, n}(\Omega)$ satisfies $\int_{\Omega}|\nabla u|^{n} d x \leq 1$, then

$$
\int_{\Omega} e^{\beta|u|^{\frac{n}{n-1}}} d x<C_{0} .
$$

The proof involves expanding $e^{\beta|u|^{\frac{n}{n-1}}}$ by its Taylor series, then applying the Sobolev inequality to each term. Trudinger's proof, however, did not give the optimal value of the constant $\beta_{n}$. This was later found by Moser [21] to be $\beta_{n}=n\left(\omega_{n-1}\right)^{\frac{1}{n-1}}$, where $\omega_{n-1}$ is the volume of the $(n-1)$ dimensional unit sphere in $\mathbb{R}^{n-1}$.

In Alice's joint work with Carleson [2], they proved a very surprising result:

Theorem 1.2 ([2]). If $\Omega=B \subset \mathbb{R}^{n}$ is the unit ball, then an extremal exists for the Moser-Trudinger inequality. That is, there is a function $u \in W_{0}^{1, n}(B)$ with $\|\nabla u\|_{L^{n(B)}}=1$ and

$$
\int_{B} e^{\beta_{n}|u|^{\frac{n}{n-1}}} d x=\sup _{\left\{v \in W_{0}^{1, n}(B):\|\nabla v\|_{n} \leq 1\right\}} \int_{B} e^{\beta_{n}|v|^{\frac{n}{n-1}}} d x .
$$

As we saw above, there are no extremals of the Sobolev inequality for functions supported on the ball. Since the Moser-Trudinger inequality follows from the Sobolev inequality (in some sense), the Carleson-Chang result was quite unexpected.

\section{First Digression: Bubbling}

Before continuing with our description of Alice's early work in geometric analysis, it will be helpful to provide more mathematical context.

The Moser-Trudinger inequality has a counterpart for functions defined on the unit sphere $S^{2}$. Let $\left(S^{2}, g_{0}\right)$ denote the unit sphere with its standard Riemannian metric. In this case the Moser-Trudinger inequality takes the form

$$
\int_{S^{2}} e^{\frac{4 \pi(u-\bar{u})^{2}}{\|\nabla u\|_{2}^{2}}} d A \leq C_{1},
$$


where $d A$ is the area form with respect to $g_{0}$ and $\bar{u}$ denotes the mean value of $u$. This implies another inequality, sometimes referred to as its "linearized" version:

$$
\log \left(\frac{1}{4 \pi} \int_{S^{2}} e^{2 u} d A\right) \leq \frac{1}{4 \pi} \int_{S^{2}}\left(|\nabla u|^{2}+2 u\right) d A+C_{0}
$$

for some constant $C_{0}$ that is independent of $u$.

Moser was interested in the sharp value of $\beta_{n}$ in part because this version of the inequality arises in a question posed by Louis Nirenberg: Given $K \in C^{\infty}\left(S^{2}\right)$, is there a conformal metric $g=e^{2 w} g_{0}$ such that the Gauss curvature of $g$ is given by $K$ ? A standard calculation shows that this question can be answered in the affirmative if and only if one can find a solution of the PDE

$$
\Delta_{0} w+K e^{2 w}=1,
$$

where $\Delta_{0}$ is the Laplace-Beltrami operator with respect to $g_{0}$.

There is an obvious necessary condition on the candidate function $K$ given by the Gauss-Bonnet formula (equivalently, by integrating (5) over the sphere): $K$ must be positive somewhere. Later, Kazdan and Warner proved that a more subtle condition must hold. However, their condition is implicit and involves not only the function $K$ but the putative solution $w$.

Moser used a variational approach to study this question, and considered the functional $J: H^{1,2}\left(S^{2}\right) \rightarrow \mathbb{R}$ given by

$$
\begin{aligned}
J_{K}[w]= & \frac{1}{4 \pi} \int_{S^{2}}\left(|\nabla w|^{2}+2 w\right) d A \\
& -\log \left(\frac{1}{4 \pi} \int_{S^{2}} K e^{2 w} d A\right) .
\end{aligned}
$$

It is not difficult to see that $w$ is a critical point of $J$; i.e.,

$$
\left.\frac{d}{d s} J_{K}[w+s \phi]\right|_{s=0}=0
$$

for all $\phi \in W^{1,2}\left(S^{2}\right)$ if and only if $w$ is a weak solution of (5). In fact, using the Sobolev embedding theorem and standard elliptic regularity, any critical point of $J$ is automatically smooth and therefore a classical solution of (5).

At this point it will be illuminating to explain one of the fundamental difficulties of the Nirenberg problem, since the same issue will appear in other contexts. To simplify, consider the case where $K \equiv 1$. The functional $J_{1}$ enjoys a natural invariance under the action of the conformal group of $S^{2}$. Namely, if $f: S^{2} \rightarrow S^{2}$ is a conformal transformation of $S^{2}$, then $f^{*} g_{0}=e^{2 \varphi_{f}} g_{0}$ for some function $\varphi_{f} \in C^{\infty}\left(S^{2}\right)$. Therefore, if $g=e^{2 w} g_{0}$, then $f^{*} g=e^{2 w_{f}} g_{0}$, where $w_{f}=w \circ f+\varphi_{f}$. One can show that

$$
J_{1}[w]=J_{1}\left[w_{f}\right] .
$$

Since the conformal group of $\left(S^{2}, g_{0}\right)$ (indeed, the round sphere of any dimension) is noncompact, this means that $J_{1}$ is invariant under the action of a noncompact group. As a consequence, $J_{1}$ fails to satisfy the Palais-Smale condition, and many standard variational methods that are used to establish the existence of critical points are inapplicable. We can see this very concretely through the following construction: If $N=(0,0,1)$ denotes the "north pole" of $S^{2} \subset \mathbb{R}^{3}$, let $\sigma: S^{2} \backslash\{N\} \rightarrow \mathbb{R}^{2}$ be the stereographic projection map (i.e., a point $P$ in $S^{2}$ is sent to the point in the plane determined by following the light ray from $N$ through $P$ ). One can check by hand that $\sigma$ is conformal: $\sigma^{*} d s^{2}=e^{2 u_{0}} g_{0}$ for some function $u_{0} \in C^{\infty}\left(S^{2}\right)$, where $d s^{2}$ is the Euclidean metric. For $\lambda>0$ let $\delta_{\lambda}: \mathbb{R}^{2} \rightarrow \mathbb{R}^{2}$ denote dilation by $\lambda^{-1}$ (i.e., $x \mapsto \lambda^{-1} x$ ). Then the map $f_{\lambda}=\sigma^{-1} \circ \delta_{\lambda} \circ \sigma: S^{2} \rightarrow S^{2}$ is conformal: $f_{\lambda}^{*} g_{0}=e^{2 u_{\lambda}} g_{0}$. Moreover, if we let $\lambda \rightarrow \infty$, then the family of conformal factors $\left\{e^{2 u_{\lambda}}\right\}_{\lambda}$ will "concentrate" (or "bubble") at the north pole while converging to zero at other points. Since these metrics arise from pulling back the round metric, $J_{1}\left[u_{\lambda}\right]=0$ for all $\lambda$.

Bubbling appears in many geometric variational problems, from harmonic maps to Yang-Mills connections. In many cases the fundamental issue is showing that the loss of compactness can only arise from bubbling when the underlying manifold is the round sphere.

\section{Prescribed Scalar Curvature and the Isospectral Problem}

Not long after her work with Carleson, Alice began what would become a long collaboration with her husband, Paul Yang. Their first joint paper, "Prescribing Gaussian curvature on $S^{2}$," was on the Nirenberg problem and appeared in 1987. For a positive function $K \in C^{\infty}\left(S^{2}\right)$, they gave two different necessary conditions for the existence of a conformal metric $g$ with Gauss curvature $K$. The statements are somewhat technical, so we will state only one of them:

Theorem 3.1 (Theorem II of [14]). Let $K$ be a positive smooth function with only nondegenerate critical points, and in addition assume that $\Delta_{0} K(p) \neq 0$, where $p$ is any critical point. Suppose there are at least two local maximum points of $K$, and at all saddle points $q$ of $K, K(q)>0$. Then $K$ is the Gauss curvature of some metric conformal to $g_{0}$.

The proof of this theorem is quite involved, but keeping in mind our explanation of the bubbling phenomenon we can give some indication of the strategy. Like the work of Moser and others, their approach was variational: that is, the goal is to show the existence of critical points of the functional $J_{K}$ in (6) via a min-max scheme. As we discussed above, due to the conformal invariance of the problem, a sequence $\left\{w_{j}\right\}$ produced by the min-max procedure may fail to be bounded in $W^{1,2}\left(S^{2}\right)$. A key step in the proof of the Chang-Yang result is showing that when this happens, $\left\{e^{2 u_{j}}\right\}$ must concentrate at a single point $p \in S^{2}$ (which we can assume is the north pole), just like the 


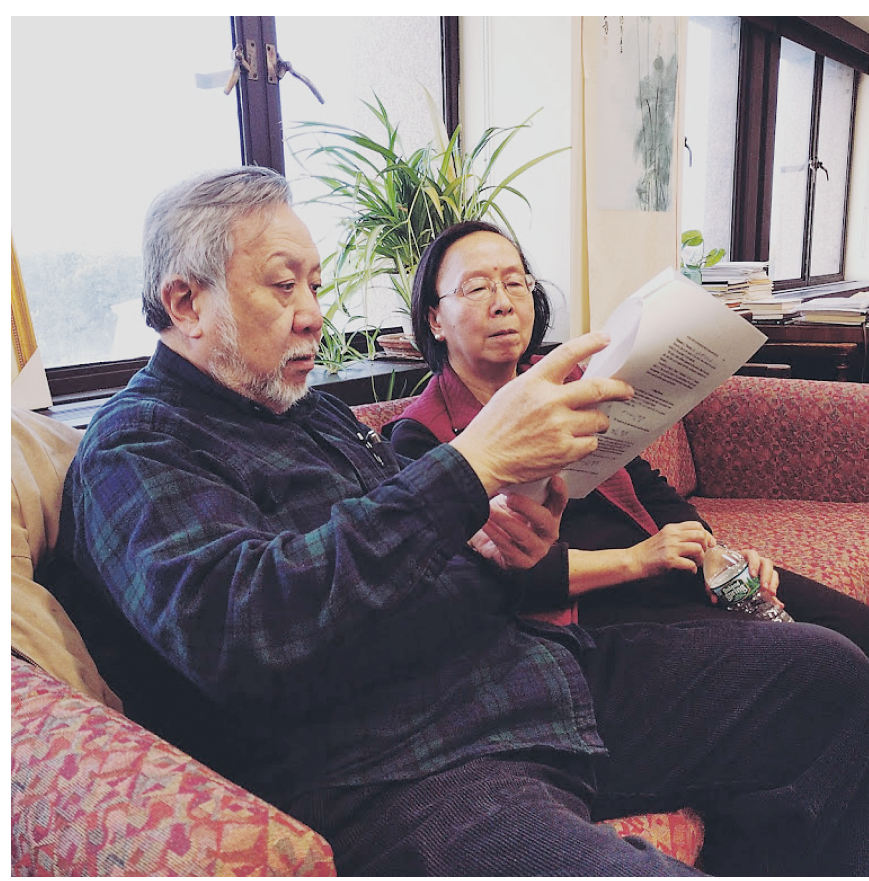

Partners in life, and often in mathematics.

family of conformal factors $\left\{e^{2 u_{\lambda}}\right\}$ we constructed above. In fact, they showed that there is a sequence of dilations $\left\{\lambda_{j}\right\}$ such that for each $j, e^{2 u_{j}}$ is "close" to $e^{2 w_{j}}$. With this information, they reduce the question of the behavior of the sequence $\left\{w_{j}\right\}$ to analyzing the behavior of the integral $\int K e^{2 w_{j}} d A$ when $e^{2 w_{j}}$ is highly concentrated, and conclude that this can only happen at a saddle point $q$ of $K$ with $\Delta_{0} K(q)<0$.

In subsequent papers Alice and Paul continued working on the Nirenberg problem and the higher-dimensional version of prescribing the scalar curvature. At about the same time they became interested in some questions in spectral geometry with obvious connections to their work. Their work on the isospectral problem and the regularized determinant, which began in the late 1980s, would continue for almost two decades and lead in a number of unexpected directions.

If $\left(M^{n}, g\right)$ is a closed, $n$-dimensional Riemannian manifold and $\Delta_{g}=g^{i j} \nabla_{i} \nabla_{j}$ is the Laplace operator with respect to $g$, then $-\Delta_{g}$ has discrete spectrum

$$
0=\lambda_{0}<\lambda_{1} \leq \lambda_{2} \leq \cdots \rightarrow \infty,
$$

where by convention the eigenvalues are counted with their multiplicities. Two Riemannian metrics are isospectral if their respective Laplace operators have the same spectrum. One theme of spectral geometry is the extent to which the spectrum of a Riemannian manifold determines the metric. For example, are two isospectral metrics isometric? Milnor showed that this is not the case, but one can still ask to what extent the spectrum influences the geometry and vice versa. For example, is the set of isospectral metrics on a fixed manifold $M$ compact? One way to approach these kinds of questions is to study geometric invariants that are built up from the spectrum.

One such invariant is the trace of the heat kernel, $h(t)=$ $\sum_{j} e^{-t \lambda_{j}}$, which has an expansion of the form

$$
h(t) \sim t^{-n / 2} \sum_{i=0}^{\infty} \alpha_{i} t^{i},
$$

where $n=\operatorname{dim} M$. An isospectral set of metrics necessarily has the same heat coefficients $\left\{\alpha_{i}\right\}$, and the first few coefficients can be explicitly computed. One consequence of these explicit formulas is that in three dimensions, a set of isospectral metrics has a uniform bound on the $L^{2}$-norm of their curvature tensors. This is fairly weak control, but by imposing further constraints on the set or by using other information derived from the spectrum, one might hope to get a compactness result.

In work of Brooks-Perry-Yang, they showed that if the isospectral metrics were in a fixed conformal class on a three-dimensional manifold $M^{3}$, and if the conformal class admits a metric of negative scalar curvature, then the family is compact in $C^{\infty}$. In a series of three papers culminating in [17], Alice and Paul were able to remove all assumptions on the conformal class and prove compactness modulo the conformal group. The qualifier is crucial: if the isospectral set is in the conformal class of the standard sphere, then the conformal group acts on the set, and once again bubbling can occur.

\section{Second Digression: \\ Regularized Determinants}

A spectral invariant that appears prominently in Alice's subsequent work in spectral geometry (both with Paul and other collaborators) is the regularized or functional determinant. Again let $\left(M^{n}, g\right)$ be a closed, $n$-dimensional Riemannian manifold, and label the eigenvalues of $-\Delta_{g}$ by $0=\lambda_{0}<\lambda_{1} \leq \lambda_{2} \leq \cdots$ counting multiplicities. The spectral zeta function of $\left(M^{n}, g\right)$ is

$$
\zeta(s)=\sum_{j=1}^{\infty} \lambda_{j}^{-s} .
$$

By Weyl's asymptotic law, $\lambda_{j} \sim j^{2 / n}$ as $j \rightarrow \infty$. Consequently, (8) defines an analytic function for $\operatorname{Re}(s)>n / 2$. If we were to assume ( 8 ) is valid near $s=0$, then we could formally differentiate to get

$$
\zeta^{\prime}(0)=-\sum_{j=1}^{\infty} \log \lambda_{j}=-\log \operatorname{det}\left(-\Delta_{g}\right) .
$$

On the other hand, Ray-Singer showed that it is possible to meromorphically continue $\zeta$, with $s=0$ as a regular point. In view of (9), we therefore $\operatorname{define} \operatorname{det}\left(-\Delta_{g}\right)=e^{-\zeta^{\prime}(0)}$. 
Since the determinant is obviously a global invariant, it is all the more remarkable that Polyakov was able to write a local formula for the ratio of the determinants for two conformal metrics on a closed surface $M^{2}$. Suppose $\hat{g}=$ $e^{2 w} g$. Then

$$
\log \frac{\operatorname{det}\left(-\Delta_{\hat{\mathrm{g}}}\right)}{\operatorname{det}\left(-\Delta_{\mathrm{g}}\right)}=-\frac{1}{12 \pi} \int_{M^{2}}\left(|\nabla w|^{2}+2 K w\right) d A,
$$

where $K=K_{\mathrm{g}}$ is the Gauss curvature of $g$. The right-hand side of this formula should be familiar-it is the leading order terms of the functional $J_{1}$ we encountered when discussing Nirenberg's problem and the Moser-Trudinger inequality! In fact, if $g=g_{0}$ is the round metric on $S^{2}$ and we fix the area of $\hat{g}$ to be $4 \pi$, then the Moser-Trudinger inequality (4) implies that

$$
\log \frac{\operatorname{det}\left(-\Delta_{\hat{g}}\right)}{\operatorname{det}\left(-\Delta_{0}\right)} \leq C_{1}
$$

Onofri showed that $C_{1}=0$ and that equality occurs if and only if $\hat{g}=f^{*} g_{0}$ for some conformal map $f: S^{2} \rightarrow S^{2}$. We therefore conclude that the round metric, and its images under the action of the conformal group, maximize the determinant. This is a beautiful example of how the spectrum of the Laplacian is intimately tied to the geometry.

For a general Riemann surface $(M, g)$ the formula (10) defines an action on the space of unit volume conformal metrics, and critical points are precisely those metrics of constant Gauss curvature. In a series of papers, OsgoodPhillips-Sarnak used the regularized determinant to study the compactness of isospectral metrics on surfaces, and the existence of extremals of the functional determinant in a fixed conformal class. The latter result gave a spectral theoretic proof of the uniformization theorem.

\section{The Regularized Determinant and Q-curvature}

In deriving (10) Polyakov exploited a crucial property of the Laplacian in two dimensions, namely, its conformal covariance: if $\hat{g}=e^{2 w} g$, then

$$
\Delta_{\hat{g}}=e^{-2 w} \Delta_{g}
$$

In higher dimensions this property fails, but there are other conformally covariant differential operators. For example, the conformal Laplacian

$$
L=-\Delta+\frac{(n-2)}{4(n-1)} R
$$

where $R$ is the scalar curvature, is conformally covariant in the sense that

$$
L_{e^{2 w} w \phi}=e^{-\frac{n+2}{2} w} L_{g}\left(e^{\frac{n-2}{2} w} \phi\right) .
$$

In four dimensions there is another example, and this operator will play a significant role in what follows. The
Paneitz operator is defined by

$$
P=(-\Delta)^{2}+\delta\left(\frac{2}{3} R g-2 R i c\right) \circ \nabla,
$$

where $\delta$ is the divergence operator and the curvature term $\frac{2}{3} R g-2 R i c$ should be viewed as an endomorphism of the tangent bundle, by acting on the gradient of a function. The Paneitz operator is named for S. Paneitz, who wrote it down in an unpublished preprint before his untimely death. However, it was actually discovered earlier by physicists. The Paneitz operator is conformally convariant in the sense that $P_{e^{2 w}}=e^{-4 w} P_{g}$.

As we shall see, in ways beyond conformal covariance this operator can be viewed as the natural generalization of the Laplace operator to four-manifolds. For example, as we saw when discussing the Nirenberg problem, the Laplace operator arises in the formula (5) relating the Gauss curvatures of conformally related metrics. In four dimensions, there is a curvature quantity, which Branson called the "Q-curvature," defined by

$$
Q=\frac{1}{12}\left(-\Delta R+R^{2}-3|R i c|^{2}\right) .
$$

Where does this strange quantity come from? One answer, which we will come to below, is topological: it appears naturally in the Chern-Gauss-Bonnet formula. But the answer that is most relevant to the current discussion is that it is the curvature quantity naturally associated to the Paneitz operator. More precisely, given two conformal metrics $\hat{g}=e^{2 w} g$, their respective $Q$-curvatures are related by the formula

$$
P w+2 Q=2 Q_{\hat{g}} e^{4 w} .
$$

The parallel with (5) is obvious. Moreover, the total $Q$ curvature is a conformal invariant:

$$
\int Q_{\hat{g}} d \hat{V}=\int Q_{g} d V .
$$

In [1], Branson and Ørsted were able to generalize Polyakov's technique to conformally covariant operators defined on a four-manifold $M^{4}$. The resulting formula, unfortunately, is much more complicated than its predecessor (10). Rather than writing it out explicitly, it may be more illuminating to give a schematic description. To this end, suppose $A=A_{g}$ is a conformally covariant operator, let $\hat{g}=e^{2 w} g$ be conformal metrics, and consider the functional

$$
\hat{\mathrm{g}} \mapsto \log \frac{\operatorname{det} A_{\hat{\mathrm{g}}}}{\operatorname{det} A_{\mathrm{g}}} .
$$

Since the determinant is not scale invariant, we add a normalizing term and consider a normalized version of the functional $F_{A}$, which satisfies $F_{A}[w+c]=F_{A}[w]$.

The first thing to note is that $F_{A}$ can always be expressed as a linear combination of three universal (i.e., independent of the particular operator) terms. Different operators 
will result in different linear combinations of these three terms. Alice and Paul introduced the convention of expressing the formula in the following way:

$$
F_{A}[w]=\gamma_{1}(A) I[w]+\gamma_{2}(A) I I[w]+\gamma_{3}(A) I I I[w],
$$

where $\left(\gamma_{1}, \gamma_{2}, \gamma_{3}\right)$ is a triple of real numbers, and $I, I I, I I I$ are the three subfunctionals, each of which has a specific geometric interpretation. The contribution of BransonØrsted was not only the general formula (17) but the calculation of the coefficients for some specific operators. For example, if $A=L$, the conformal Laplacian, then $\gamma_{1}(L)=1$, $\gamma_{2}(L)=-4$, and $\gamma_{3}(L)=-2 / 3$.

Alice and Paul showed that each of the functionals $I, I I$, and $I I I$ is the Lagrangian associated to a natural geometric variational problem:

$$
\hat{g}=e^{2 w} g \text { is a critical point of } I \Longleftrightarrow\left|W_{\hat{g}}\right|^{2}=\text { const., }
$$

where $W$ denotes the Weyl tensor;

$$
\begin{gathered}
\hat{g}=e^{2 w} g \text { is a critical point of } I I \Longleftrightarrow Q_{\hat{g}}=\text { const.; } \\
\hat{g}=e^{2 w_{g}} \text { is a critical point of } I I I \Longleftrightarrow \Delta_{\hat{g}} R_{\hat{g}}=0 .
\end{gathered}
$$

Note that critical points of $I I I$ correspond to metrics of constant scalar curvature and are therefore related to the Yamabe problem. Using these formulas, we see that a metric $\hat{g}=e^{2 w} g$ is a critical point of $F_{A}$ if and only if the curvature of $\hat{g}$ satisfies

$$
\gamma_{1}\left|W_{\hat{g}}\right|^{2}+\gamma_{2} Q_{\hat{g}}-\gamma_{3} \Delta_{\hat{g}} R_{\hat{g}}=\text { const. }
$$

If we express this in terms of the conformal factor $w$, it is a fourth-order semilinear PDE with leading term $\Delta^{2} w$.

In joint work with Branson, Alice and Paul studied $F_{A}$ in the conformal class of the round sphere $\left(S^{4}, g_{0}\right)$ when $A=$ $L$, the conformal Laplacian. They showed that all three functionals $-I, I I$, and $I I I$-are minimized by the round metric, up to conformal transformations. In particular, $\operatorname{det} L_{g_{0}} \leq \operatorname{det} L_{g}$ for all conformal metrics $g=e^{2 w} g_{0}$.

After the work with Branson, Alice and Paul wrote a seminal paper, "Extremal metrics of zeta function determinants on 4-manifolds," which appeared in Annals of Mathematics [15]. In addition to giving general existence results for extremals of the determinant, it also was the first paper to study the $Q$-curvature equation and illustrate the connection between the variational properties of the functional $I I$ and the kernel of the Paneitz operator. Subsequently, the first author of the current article observed that some of the technical assumptions in [15] could be dropped in certain cases, so to avoid introducing additional notation we will give a simplified version of one of the main existence results in [15]:

Theorem 5.1. If the scalar curvature of $\left(M^{4}, g\right)$ is positive, then there is a metric $\hat{g}=e^{2 w}$ g that extremizes $\operatorname{det} L$, where $L$ is the conformal Laplacian. If $\chi\left(M^{4}\right) \leq 0$, then the extremal metric is unique.
In the case of surfaces of higher genus the extremal of the determinant of the Laplacian is also known to be unique, so we have a beautiful parallel between the twoand four-dimensional pictures. However, it is also important to emphasize that the functional determinant is more nonlinear in four dimensions, and there are phenomena that have no parallel with the two-dimensional case. In fact only recently has progress been made on the existence of critical points of $F_{A}$ when the scalar curvature of $\left(M^{4}, g\right)$ is negative.

For the functional $I I$, Chang-Yang gave the first general existence result for metrics with constant $Q$-curvature:

Theorem 5.2. If the total Q-curvature $\int_{M^{4}} Q_{g} d V$ is less than that of the round sphere, and the Paneitz operator $P_{g}$ is positive with trivial kernel, then there is a conformal metric $\hat{g}$ with constant Q-curvature.

The proof consisted of showing that the functional $I I$ can be minimized. They also showed that the triviality of the kernel was a necessary condition for II to have a lower bound.

Over the next several years Alice and Paul would continue working on the analytic and geometric aspects of the $Q$-curvature. In [16] they proved Liouville-type theorems for entire solutions. In beautiful work with J. Qing ([11], [10]), they studied the total Q-curvature of complete, locally conformally flat (LCF) four-manifolds.

\section{Fully Nonlinear Equations}

In the late 1990s two developments in conformal geometry would lead to Alice's interest in fully nonlinear equations. The first arose from her work with Paul on the functional determinant. If the constants $\gamma_{i}$ in (17) are chosen so that $\gamma_{2}+12 \gamma_{3}=0$, then it turns out that the highest-order terms in the Euler equation (18) cancel, and the equation becomes a second-order equation in the metric. If we further take $\gamma_{1}=0$ to eliminate the Weyl tensor, then (18) simplifies to

$$
-3\left|R i c_{\hat{g}}\right|^{2}+R_{\hat{g}}^{2}=\kappa,
$$

where $x$ is a constant. The significance of this is the following: if the constant $x>0$, and the scalar curvature of the critical metric $\hat{g}=e^{2 w} g$ is positive, then a simple calculation shows that the Ricci curvature of $\hat{g}$ must be positive. This suggests the idea of finding critical points of $F_{A}$ in order to construct metrics with positive Ricci curvature. This was carried out by Alice in joint work with Paul and the first author of this article in [8]. It will be easier to state the main result of that paper if we first describe the concurrent development in conformal geometry. 
Given an $n$-dimensional Riemannian manifold $(M, g)$, one can decompose the curvature tensor as Riem $=W+$ $A \wedge g$, where $A$ is the Schouten tensor,

$$
A=\frac{1}{n-2}\left(R i c-\frac{1}{2(n-1)} R g\right)
$$

and $\wedge$ is the exterior product extended to symmetric 2 -tensors (viewed as $T^{*} M$-valued one-forms and often referred to as the Kulkarni-Nomizu product). Since the Weyl tensor is conformally invariant, the behavior of the curvature tensor under a conformal change of metric is determined by the Schouten tensor. In his thesis, Jeff Viaclovsky initiated the study of the functionals

$$
g \mapsto \int \sigma_{k}\left(g^{-1} A_{g}\right) d V,
$$

restricted to the space of unit volume conformal metrics $[g]_{1}=\left\{\hat{g}=e^{2 w} g, \operatorname{Vol}(\hat{g})=1\right\}$. Here we are using the convention that $\left(g^{-1} A\right)_{i}^{j}=g^{j k} A_{i k}$. When $k=1$, up to a multiple the functional (21) is just the total scalar curvature, and critical points are metrics with constant scalar curvature. When $k>1$ the situation is more complicated. If $k=2$ but $n \neq 4$, then the same holds: a metric $\hat{g} \in[g]_{1}$ is a critical point if and only if

$$
\sigma_{2}\left(\hat{\mathrm{g}}^{-1} A_{\hat{\mathrm{g}}}\right)=\text { const. }
$$

When $k=2$ and $n=4$ the integral in (21) is conformally invariant. In fact, $\sigma_{2}$ is related to the $Q$-curvature:

$$
Q=-\frac{1}{12} \Delta R+2 \sigma_{2}\left(g^{-1} A\right)
$$

so the total $Q$-curvature (a conformal invariant) is just a multiple of the total $\sigma_{2}$-curvature.

When $k>2$ but $k \neq n / 2$, and if we assume the conformal structure is locally conformally flat, then critical points of (21) satisfy

$$
\sigma_{k}\left(\hat{\mathrm{g}}^{-1} A_{\hat{\mathrm{g}}}\right)=\text { const. }
$$

In fact, Branson and Gover showed that conformal flatness is a necessary condition; otherwise additional terms will appear in the Euler-Lagrange equation (21).

Viaclovsky proposed the problem of solving the equations (24) (whether they arise variationally or not) as a generalization of the Yamabe problem. They are now referred to as the " $\sigma_{k}$-curvature equations," and the problem of finding solutions as the $\sigma_{k}$-Yamabe problem. Since they are fully nonlinear, one has to impose an ellipticity condition. Although there is now an extensive existence theory for solutions, some basic questions (especially about regularity) remain unanswered.

The connection between the study of the functional determinant in dimension four and the equations introduced by Viaclovsky is the following: the special case of the Euler equation (19) is, up to a constant, the equation (22).
Using the determinant functional, Chang-Gursky-Yang proved

Theorem 6.1 ([8], [7]). Let $\left(M^{4}, g\right)$ be a closed Riemannian four-manifold with positive scalar curvature. If

$$
\int_{M^{4}} \sigma_{2}\left(g^{-1} A_{g}\right) d V>0
$$

then there is a smooth conformal metric $\hat{g}=e^{2 w}$ g solving (22). Moreover, the Ricci curvature of $\hat{\mathrm{g}}$ satisfies $0<R i c_{\hat{\mathrm{g}}}<\frac{1}{2} R_{\hat{\mathrm{g}}} \hat{\mathrm{g}}$.

This was the first existence result for the $\sigma_{k}$-Yamabe problem. Moreover, since the conditions are conformally invariant, it is relatively easy to construct examples of manifolds satisfying the hypotheses. Later, Chang-GurskyYang would use a version of the $\sigma_{2}$-curvature equation in which the right-hand side includes the norm of the Weyl tensor in order to prove a conformally invariant sphere theorem.

When $k=n$ the $\sigma_{k}$-curvature equation shares many features with a special case of the equations studied in the theory of optimal transportation. In joint work of Alice with the second author ([12], [13]), they proved quermassintegral inequalities for a large class of nonconvex domains.

In convex geometry, the quermassintegral inequalities are a family of inequalities comparing the mixed volumes $V_{k}(\Omega)$ of a convex body $\Omega \subset \mathbb{R}^{n}$. If $\Omega$ has a smooth boundary, then the mixed volumes are the intrinsic counterparts of the functionals introduced by Viaclovsky:

$$
V_{n-k-1}(\Omega)=c_{k, n} \int_{\partial \Omega} \sigma_{k}(L) d \mu
$$

where $L$ is the second fundamental form of $\partial \Omega$. The quermassintegral inequalities can be derived as a consequence of the classical Alexandrov-Fenchel inequalities. For a general nonconvex domain, one does not expect the quermassintegral (or Alexandrov-Fenchel) inequalities to hold. Chang-Wang proved that $(k+1)$-convex domains, which contain a large class of nonconvex domains (for example, "thin" tori) still satisfy the $k$ th of quermassintegral inequality.

\section{Conformally Compact Einstein Manifolds}

The final area of Alice's oeuvre we will attempt to summarize includes some of her most recent work. If $X$ is a compact manifold of dimension $n+1$ with nonempty boundary $M=\partial X$, a metric $g$ defined in the interior of $X$ is called conformally compact if there is a defining function for the boundary (i.e., a function $\rho: X \rightarrow \mathbb{R}$ such that $\rho>0$ in $X$, $\rho=0$ on $\partial X$, and $d \rho \neq 0$ on $\partial X$ ) such that $\rho^{2} g$ extends to a Riemannian metric on $X$. If $|d r|_{g}=1$ on $\partial X$, then one can show that the sectional curvatures of $(X, g)$ tend to -1 at infinity, and we say that $(X, g)$ is asymptotically hyperbolic.

If $g$ is an Einstein metric, then $(X, g)$ is a PoincaréEinstein manifold (or CCE manifold, for "conformally 
compact Einstein"). The canonical example of a CCE manifold is the Poincaré ball model of hyperbolic space: in this case $X=\bar{B}^{n+1}$ is the unit ball in $\mathbb{R}^{n+1}$, and $g=$ $4\left(1-|x|^{2}\right)^{-2} d x^{2}$ is the hyperbolic metric. Notice that we can take $\rho(x)=\left(1-|x|^{2}\right) / 2$ as a defining function, and $\rho^{2} g=d x^{2}$ is the Euclidean metric. It is easy to see that CCE metrics are asymptotically hyperbolic.

The defining function of a conformally compact metric is not unique, and the compactified metrics defined by two different choices of defining function are conformal. Therefore, one can associate to any conformally compact metric $g$ defined in the interior of $X$ a conformal class of metrics on the compact manifold $X$, and by restricting to the boundary a conformal class of metrics $\left[h=\left.\rho^{2} g\right|_{M}\right]$ on $M=\partial X$. We call $[h]$ the conformal infinity of $g$. For example, if $(X, g)$ is the hyperbolic metric as above, then the boundary conformal class defined in this way is the conformal class of the Euclidean metric restricted to $S^{n}=\partial B(0,1)^{n+1}$, i.e., the conformal class of the round metric.

CCE manifolds appear in the Fefferman-Graham theory of conformal invariants [18] and in the AdS/CFT correspondence in theoretical physics. They have sometimes unexpected connections to other areas of mathematics, including some of the topics mentioned above. Alice's first results in the subject were joint with Paul and Jie Qing and studied the connection between an important invariant of CCE manifold $(X, g)$, known as the renormalized volume, and the topology of $X$.

Given a CCE metric we can always construct a defining function $r$ for which $|d r| \equiv 1$ near $\partial X$. Using the Fefferman-Graham expansions, for $\epsilon>0$ the volume of the region $X_{\epsilon}=\{x \in X \quad: \quad r(x)>\epsilon\}$ has an expansion in terms of $\epsilon$ depending on the parity of the dimension. If $n$ is odd, then

$$
\begin{aligned}
\operatorname{Vol}\left(X_{\epsilon}\right)= & c_{0} \epsilon^{-n}+c_{2} \epsilon^{-n+2}+\text { (odd powers) } \\
& +c_{n-1} \epsilon^{-1}+V+o(1),
\end{aligned}
$$

where the $c_{i}$ 's are given by integrals of polynomials in the curvature of the induced metric $h=\left.r^{2} g\right|_{M}$ on the boundary. If $n$ is even, a logarithmic term appears; to simplify the exposition we will discuss only the odd-dimensional case.

Remarkably, the constant term $V$ in the expansion (26) is independent of the choice of defining function, and is therefore a conformal invariant of the boundary (see [19]). When $n=3$, in [9] Chang-Qing-Yang showed that if

$$
V>\frac{1}{3} \frac{4 \pi^{2}}{3} \chi(X)
$$

then $X$ is homeomorphic to $B^{4}$, up to a possible finite cover. Moreover, if the constant on the right is improved from $1 / 3$ to $1 / 2$, then $X$ is diffeomorphic to $B$ and $\partial X$ is diffeomorphic to $S^{3}$. Two of the ingredients of their proof are the sphere theorem of Chang-Gursky-Yang mentioned above and a formula when $n=3$ due to Anderson that relates $V$ to the Euler characteristic and the $L^{2}$-norm of the Weyl tensor.

In subsequent work, Chang-Qing-Yang showed that Anderson's formula is actually a special case of a much more general formula for $n$ odd that expresses the renormalized volume of a CCE manifold in terms of the Euler characteristic and a conformally invariant integral. The integrand is a sum of contractions of the Weyl tensor and its covariant derivatives; when $n=5$ they gave an explicit formula.

In addition to the conformal Laplacian and Paneitz operator there are other examples of conformally covariant operators. Graham-Zworksi used scattering theory methods on asymptotically hyperbolic manifolds to construct families of conformally invariant operators $P_{\gamma}$ with principal symbol the same as $(-\Delta)^{\gamma}$ for $\gamma \in(0, n)$. When $\gamma=k \in(0, n / 2)$ is an integer, $P_{k}$ corresponds to the operators found earlier by Graham-Jenne-Mason-Sparling, but for noninteger values their construction gives examples of conformally covariant nonlocal operators.

Fractional powers of the Laplacian also appear in various mathematical models of physical phenomena. On Euclidean space one can use the Fourier transform to define $(-\Delta)^{\gamma}$, but Caffarelli-Silvestre showed that for $\gamma \in(0,1)$ one can construct these operators by solving a degenerate elliptic extension problem on the upper half-space $\mathbb{R}_{+}^{n+1}$, then restricting the solution to the boundary. In joint work of Chang with Maria del Mar Gonzalez [6], they had the beautiful insight that the Graham-Zworski construction and the Caffarelli-Silvestre construction were really the same idea in different guises: properly interpreted, the Caffarelli-Silvestre extension problem was the scattering operator on the upper half-space model of hyperbolic space. This realization allowed them to recast the Caffarelli-Silvestre construction in the framework of CCE manifolds and extend the construction to $\gamma \in(0, n / 2)$. In subsequent work with Jeffrey Case they gave an alternate construction of the Graham-Zworski operators $P_{\gamma}$ and showed that under natural geometric conditions $P_{\gamma}$ satisfies a strong maximum principle. The study of fractional order problems in geometry and in applied mathematics is a highly active area, and the Chang-Gonzalez paper provided a unified way of defining and understanding many of the properties of these operators.

\section{Epilogue}

Among Alice's many professional distinctions was her selection to deliver the Emmy Noether Lecture at the International Congress of Mathematicians in 2018. The citation for the award includes a brief list of her honors: recipient of the Ruth Lyttle Satter Prize from the AMS in 1995; 
Fellow of the American Academy of Arts and Sciences since 2008; Member of the National Academy of Sciences of the USA since 2009; Academician of the Academia Sinica, Republic of China, since 2012; Doctor Honoris Causa from the Université Pierre et Marie Curie in 2013. Alice has also served the profession in many roles, including a term as department chair at Princeton. But beyond her mathematical contributions, Alice has had a passionate commitment to her students (as we can personally attest), and her influence goes beyond her mathematical progeny. "I can personally testify to the importance of having role models and the companionship of other women colleagues," Alice wrote in her response to receiving the Satter Prize. Throughout her distinguished career Alice has been such a role model: a dedicated researcher, teacher, mentor, and advocate for the profession.

\section{References}

[1] Thomas P. Branson and Bent Ørsted, Explicit functional determinants in four dimensions, Proc. Amer. Math. Soc. 113 (1991), no. 3, 669-682. MR1050018

[2] Lennart Carleson and Sun-Yung A. Chang, On the existence of an extremal function for an inequality of J. Moser (English, with French summary), Bull. Sci. Math. (2) 110 (1986), no. 2, 113-127. MR878016

[3] Sun-Yung A. Chang and Robert Fefferman, A continuous version of duality of $H^{1}$ with $B M O$ on the bidisc, Ann. of Math. (2) 112 (1980), no. 1, 179-201. MR584078

[4] Sun Yung A. Chang, A characterization of Douglas subalgebras, Acta Math. 137 (1976), no. 2, 82-89. MR428044

[5] Sun-Yung A. Chang, Carleson measure on the bi-disc, Ann. of Math. (2) 109 (1979), no. 3, 613-620. MR534766

[6] Sun-Yung Alice Chang and María del Mar González, Fractional Laplacian in conformal geometry, Adv. Math. 226 (2011), no. 2, 1410-1432. MR2737789

[7] Sun-Yung A. Chang, Matthew J. Gursky, and Paul Yang, An a priori estimate for a fully nonlinear equation on fourmanifolds, J. Anal. Math. 87 (2002), 151-186. MR1945280

[8] Sun-Yung A. Chang, Matthew J. Gursky, and Paul C. Yang, An equation of Monge-Ampère type in conformal geometry, and four-manifolds of positive Ricci curvature, Ann. of Math. (2) 155 (2002), no. 3, 709-787. MR1923964

[9] Sun-Yung A. Chang, Jie Qing, and Paul Yang, On the topology of conformally compact Einstein 4-manifolds, Noncompact problems at the intersection of geometry, analysis, and topology, Contemp. Math., vol. 350, Amer. Math. Soc., Providence, RI, 2004, pp. 49-61. MR2082390

[10] Sun-Yung A. Chang, Jie Qing, and Paul C. Yang, Compactification of a class of conformally flat 4-manifold, Invent. Math. 142 (2000), no. 1, 65-93. MR1784799

[11] Sun-Yung A. Chang, Jie Qing, and Paul C. Yang, On the Chern-Gauss-Bonnet integral for conformal metrics on $\mathbf{R}^{4}$, Duke Math. J. 103 (2000), no. 3, 523-544. MR1763657

[12] Sun-Yung Alice Chang and Yi Wang, Inequalities for quermassintegrals on k-convex domains, Adv. Math. 248 (2013), 335-377, MR3107515
[13] Sun-Yung A. Chang and Yi Wang, Some higher order isoperimetric inequalities via the method of optimal transport, Int. Math. Res. Not. IMRN 24 (2014), 6619-6644. MR3291634

[14] Sun-Yung Alice Chang and Paul C. Yang, Prescribing Gaussian curvature on $S^{2}$, Acta Math. 159 (1987), no. 3-4, 215-259. MR908146

[15] Sun-Yung A. Chang and Paul C. Yang, Extremal metrics of zeta function determinants on 4-manifolds, Ann. of Math. (2) 142 (1995), no. 1, 171-212. MR1338677

[16] Sun-Yung A. Chang and Paul C. Yang, On uniqueness of solutions of $n$th order differential equations in conformal geometry, Math. Res. Lett. 4 (1997), no. 1, 91-102. MR1432813

[17] Sun-Yung A. Chang and Paul C.-P. Yang, Isospectral conformal metrics on 3-manifolds, J. Amer. Math. Soc. 3 (1990), no. 1, 117-145. MR1015647

[18] Charles Fefferman and C. Robin Graham, The ambient metric, Annals of Mathematics Studies, vol. 178, Princeton University Press, Princeton, NJ, 2012. MR2858236

[19] C. Robin Graham, Volume and area renormalizations for conformally compact Einstein metrics, The Proceedings of the 19th Winter School "Geometry and Physics" (Srní, 1999), Rend. Circ. Mat. Palermo (2) Suppl. 63 (2000), 31-42. MR1758076

[20] Donald E. Marshall, Subalgebras of $L^{\infty}$ containing $H^{\infty}$, Acta Math. 137 (1976), no. 2, 91-98. MR428045

[21] J. Moser, A sharp form of an inequality by N. Trudinger, Indiana Univ. Math. J. 20 (1970/71), 1077-1092. MR301504

[22] Neil S. Trudinger, On imbeddings into Orlicz spaces and some applications, J. Math. Mech. 17 (1967), 473-483. MR0216286

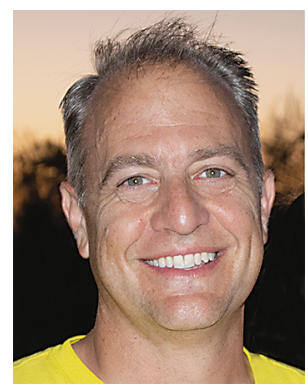

Matthew J. Gursky

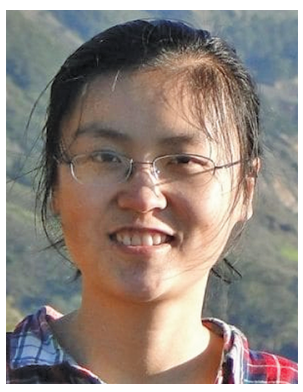

Yi Wang

Credits

Opening photo is from the video Inspiring Generations: Alice Chang. Reproduced by permission of Nick Donnoli Productions, LLC.

Photo of Sun-Yung Alice Chang and Paul Yang is courtesy of Lusann Yang.

Author photos are courtesy of the authors. 